Pacific Journal of Mathematics

ON THE ITERATES OF DERIVATIONS OF PRIME RINGS

SMith Martingale, III And C. Robert Miens 


\section{ON THE ITERATES OF DERIVATIONS OF PRIME RINGS}

\section{W. S. MARTindale, III ANd C. Robert Miers}

In this paper we study properties of associative derivations whose iterates are related in rather special ways to the original derivation, or to the iterates of another derivation. An associative derivation $d: R \rightarrow R$ is an additive (or linear when appropriate) mapping on a ring $R$ satisfying $d(x y)=x d(y)+d(x) y$ for all $x, y \in R$. A derivation $d: R \rightarrow R$ is called inner if $d(x)=(\operatorname{ad} a)(x)$ for some $a \in R$ where $(\operatorname{ad} a)(x)=$ $[a, x]=a x-x a$. In particular we ask when can the iterate of an inner derivation be an inner derivation? When can the iterates of two derivations commute? More precisely, we characterize elements $a, b \in R, R$ a prime ring, for which $(\operatorname{ad} a)^{n}(x)=(\operatorname{ad} b)(x)$ for all $x \in R$, and we characterize derivations $d: R \rightarrow R, \delta: R \rightarrow R$ for which $\left[d^{n}(x), \delta^{n}(y)\right]$ $=0$ for all $x, y \in R, R$ prime. Applications are made to $C^{*}$-algebras.

1. Introduction. In [15] it was shown that if $d_{1}$ and $d_{2}$ are derivations of a prime ring not of characteristic 2 with $d_{1} \circ d_{2}$ a derivation, then either $d_{1}=0$ or $d_{2}=0$. Consequently if $d^{2}(x)=0$ for all $x, d$ a derivation on such a ring, then $d=0$. In [6] it was shown that if $(\operatorname{ad} a)^{n}(x)=0$ for all $x$ in a simple ring there exists a scalar $\lambda$ such that $(a-\lambda e)^{[(n+1) / 2]}$ $=0$. And in [14] it was shown that if $(\operatorname{ad} a)^{3}(x)=(\operatorname{ad} a)(x)$ for a self-adjoint $a$ and all $x$ in a von Neumann algebra then $(a-z)^{2}=a-z$ for some central element $z$. In [7] it was shown that if $[d(x), d(y)]=0$ for all $x, y \in R$ where $R$ is a prime ring and char $R \neq 2$, then $d=0$ or $R$ is a commutative integral domain. Our results show that if $d$ and $\delta$ are as above, and $R$ is prime of characteristic 0 then $R$ is commutative, or $d^{3 n-1}=0$, or $\delta^{3 n-1}=0$. If $d=\delta=\operatorname{ad} b$ there exists $\lambda$ in the extended centroid of $R$ such that $a=b-\lambda$ satisfies $a^{[(2 n+3) / 3]}=0$.

In $\S \S 2$ and 3 we prove results in the full generality of prime rings. Crucial use is made of the notions of extended centroid and central closure of a prime ring and of a key result on tensor products of closed prime algebras. We summarize these constructions by quoting from [11]. Let $R$ be a prime ring and let $T$ be the totality of all right $R$-homomorphisms $f: U_{R} \rightarrow R_{R}$, where $U$ ranges over the non-zero ideals of $R$. An equivalence relation $\sim$ is defined on $T$ as follows: $f$ (acting on $U$ ) $\sim g$ (acting on $V$ ) if $f=g$ on $W$ where $W$ is non-zero ideal contained in $U \cap V$. The set $Q=\{\hat{f}\}$ of all equivalence classes forms a ring under the operations induced by addition and composition of representatives of the equivalence classes. $R \subseteq Q$ via the map $a \rightarrow \hat{a}_{l}$ where $a_{l}$ is left multiplication by $a$ acting on $R$. The center $C$ of $Q$ is a field containing the centroid 
of $R$ and is called the extended centroid of $R$. The $C$-algebra $A=R C+C$ is again a prime ring and is called the central closure of $R$. In general we define a prime algebra $S$ over a field $F$ to be a closed prime algebra over $F$ if $S$ is its own central closure, i.e., the extended center of $S$ is just $F$ itself. We list some examples of closed prime algebras which are important for the purposes of this paper:

(1) The central closure of a prime ring ([12], p. 503).

(2) $A \otimes_{C} F$, where $A$ is a closed prime algebra over $C$ and $F$ is an extension field of $C([2]$, Theorem 3.5).

(3) Any 2-fold transitive algebra of linear transformations on a complex vector space ([4], Theorem 2.1.3 and [11], Theorem 12).

Finally, if $A$ is an algebra over $F$ we denote by $A_{l}$ the algebra of left multiplications $a_{l}$ of $A$ determined by the elements of $A$ and $A_{r}$, the algebra of right multiplications $a_{r}$ determined by the elements of $A$. A key result on tensor products is

THEOREM 1. If $S$ is a closed prime algebra over $F$ and $S^{0}$ is the opposite algebra of $S$ then

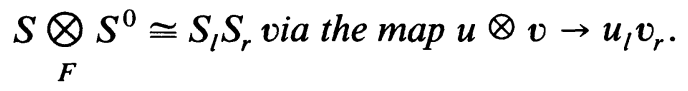

In $\S 4$ the results of $\S \S 2$ and 3 are applied to $C^{*}$-algebras. Although $C^{*}$-algebras, in general, are not prime they have a complete set of (algebraically) irreducible representations and, in our case, a phenomenon which occurs in each of these representations can be translated to a corresponding result for the original algebra. For a full account of $C^{*}$-algebras we refer the reader to [1].

\section{Iterates of an inner derivation.}

LEMMA 1. Let $R$ be a prime ring of characteristic $>n>1$, and suppose $(\mathrm{ad} a)^{n}=\mathrm{ad} b$ for some $a, b \in R$. Let $C$ be the extended centroid of $R$, $A=R C+C$ the central closure of $R, F$ the algebraic closure of $C$, and $S=A \otimes_{C} F$. Then $a$ is algebraic over $F$ of degree $\leq n$, and if $p(x)=$ $(x-\lambda)^{\prime} g(x)$ is the minimum polynomial of a over $F$ where $\lambda \in F$ is a root of multiplicity $l \geq 1$, then $b-\beta=c^{n}, \beta \in F$, and

$$
\sum_{k=0}^{n}(-1)^{k}\left(\begin{array}{l}
n \\
k
\end{array}\right) c^{n-k} \otimes c^{k}=c^{n} \otimes 1-1 \otimes c^{n}
$$

holds in $S \otimes_{F} S^{0}$ with $c=a-\lambda$. 
Proof. The condition $(\operatorname{ad} a)^{n}=\operatorname{ad} b$ clearly lifts to $S$, i.e., $\left(a_{l}-a_{r}\right)^{n}=b_{l}-b_{r}$ holds in the algebra $S_{l} S_{r}$. By Theorem 1, $S \otimes_{F} S^{0} \cong$ $S_{l} S_{r}$ via $u \otimes v \rightarrow u_{l} u_{r}$ since $S$ is a closed prime algebra over $F$, and the condition further translates to

$$
\sum_{k=0}^{n}(-1)^{k}\left(\begin{array}{l}
n \\
k
\end{array}\right) a^{n-k} \otimes a^{k}=b \otimes 1-1 \otimes b \text { in } S \otimes S_{F}^{0} .
$$

It is clear that $b \in \operatorname{span}\left\{1, a, \ldots, a^{n}\right\}$. Writing $b=\sum_{i=0}^{n} \beta_{i} a^{i}, \beta_{i} \in F$, and substituting this into (2) we see that

$$
\begin{aligned}
\left(a^{n}-b+\right. & \left.\beta_{0}\right) \otimes 1 \\
& +\left(\beta_{n-1}+(-1)^{n-1} n a\right) \otimes a^{n-1}+\left(\beta_{n}+(-1)^{n}\right) \otimes a^{n}=0 .
\end{aligned}
$$

If $1, a, \ldots, a^{n}$ are independent then, in particular, $\beta_{n-1}+(-1)^{n-1} n a=0$, whence $a= \pm \beta_{n-1} / n \in F$ a contradiction. Therefore $\left\{1, a, \ldots, a^{n}\right\}$ is a dependent set and we have established that $a$ is algebraic over $F$ of degree $\leq n$. Hence we may set $p(x)=(x-\lambda)^{l} g(x)$ as indicated in the statement of the lemma. Setting $c=a-\lambda$ we then see that $m(x)=$ $p(x+\lambda)=x^{l} q(x)$ is the minimum polynomial of $c$ over $F$ with $\operatorname{deg} m(x)=\operatorname{deg} p(x) \leq n$ and $q(0) \neq 0$. Since ad $a=\operatorname{ad} c$ we may rewrite (2) as

$$
\sum_{k=0}^{n}(-1)^{k}\left(\begin{array}{l}
n \\
k
\end{array}\right) c^{n-k} \otimes c^{k}=b \otimes 1-1 \otimes b
$$

We set $v=c^{l-1} q(c) \neq 0$, note that $c v=0$, and multiply (4) on the right by $1 \otimes v$ to obtain

$$
c^{n} \otimes v=b \otimes v-1 \otimes \beta v,
$$

whence $\left(c^{n}-b+\beta\right) \otimes v=0$. Thus $b=c^{n}+\beta$ and (4) becomes

$$
\sum_{k=0}^{n}(-1)^{k}\left(\begin{array}{l}
n \\
k
\end{array}\right) c^{n-k} \otimes c^{k}=c^{n} \otimes 1-1 \otimes c^{n}
$$

which completes the proof of the lemma.

In the following Theorem, $C, A=R C+C$ and $S=A \otimes_{C} F$ have the same meaning as in Lemma 1.

THEOREM 2. Let $R$ be a prime ring of char. $>n>1$, and suppose $(\operatorname{ad} a)^{n}=\operatorname{ad} b$ for some $a, b \in R$. If the minimum polynomial $p(x)$ of $a$ over $F$ (which necessarily exists in view of Lemma 1) contains a root $\lambda \in F$ of multiplicity $l>1$, then $\lambda \in C$ and $(a-\lambda)^{[(n+1) / 2]}=0$. 
Proof. By Lemma 1 we have

$$
\sum_{k=0}^{n}(-1)^{k}\left(\begin{array}{l}
n \\
k
\end{array}\right) c^{n-k} \otimes c^{k}=c^{n} \otimes 1-1 \otimes c^{n}
$$

holding in $S \otimes_{F} S^{0}$, where $c=a-\lambda$ and the minimum polynomial of $c$ over $F$ is $m(x)=x^{l} q(x), l>1, q(0) \neq 0$. We remark that $q(c)$, $c q(c), \cdots, c^{l-1} q(C)$ are $F$-independent. Indeed, one would have a dependency $a_{i_{1}} c^{i_{1}} q(c)+\cdots+a_{i_{r}} c^{i_{r}} q(c)=0, \alpha_{i_{1}} \neq 0, i_{1}<i_{2}<\cdots<i_{r}<l$, with "length" $r>1$ minimal. Multiplication of the dependency by $c^{l-i_{r}}$ would result in a dependency of shorter "length", a contradiction.

Now we multiply (1) on the right by $1 \otimes q(c)$ to obtain

$$
\sum_{k=0}^{l-1}(-1)^{k}\left(\begin{array}{l}
n \\
k
\end{array}\right) c^{n-k} \otimes c^{k} q(c)=0 .
$$

By the preceding remark $\left\{c^{k} q(c) \mid k=1, \cdots, l-1\right\}$ is an independent set so in particular $c^{n-(l-1)}=0$. Thus $c$ is nilpotent, with $m(x)=x^{l}$ where $l \leq n-(l-1)$, i.e., $l \leq(n+1) / 2$, and so $c^{[(n+1) / 2]}=0$. In terms of $a$ this says that $(a-\lambda)^{[(n+1) / 2]}=0$ and $p(x)=(x-\lambda)^{l}$.

It remains to prove that $\lambda \in C$. Since $c=a \otimes 1-1 \otimes \lambda \in A \otimes_{C} F$ (when written more precisely we see from $p(x)=(x-\lambda)^{l}$ that $0=$ $(a \otimes 1-1 \otimes \lambda)^{l}=\sum_{k=0}^{l}(-1)^{k}\left(\begin{array}{c}l \\ k\end{array}\right) a^{l-k} \otimes \lambda^{k}$ holds in $A \otimes_{C} F$. It follows that $a$ is algebraic over $C$ of degree $\leq l$. Let $h(x)$ be the minimum polynomial of $a$ over $C$. On the one hand we must have $\operatorname{deg} h(x) \leq$ $\operatorname{deg} p(x)=l$, and so $h(x)=p(x)$. Therefore the coefficients of $p(x)$ lie in $C$ and so from $p(x)=(x-\lambda)^{l}=x^{l}-l \lambda x^{l-1}+\cdots$ we have $l \lambda=$ $\alpha \in C$, whence $\lambda=\alpha / l \in C$.

COROLlaRY 1. Let $R$ be a prime ring of char. $>n>1$, and suppose $(\operatorname{ad} a)^{n}=$ ad $b$ for some $a, b \in R$. Then $(a-\lambda)^{[(n+1) / 2]}=0$ for some $\lambda$ in the extended centroid $C$ if either of the following conditions hold:

(a) $n$ is even;

(b) $b=0$.

Proof. By Lemma 1 we have

$$
\sum_{k=0}^{n}(-1)^{k}\left(\begin{array}{l}
n \\
k
\end{array}\right) c^{n-k} \otimes c^{k}=c^{n} \otimes 1-1 \otimes c^{n}
$$

where $c=a-\lambda$ has minimum polynomial $m(x)=x^{l} q(x), q(0) \neq 0$. We show that if either (a) or (b) holds then $c$ is nilpotent whence the conclusion follows from Theorem 2 , since then $m(x)=x^{l}$ and $l>1$. If we are given (a) then (8) becomes

$$
\sum_{k=0}^{n-1}(-1)^{k}\left(\begin{array}{l}
n \\
k
\end{array}\right) c^{n-k} \otimes c^{k}=-2 \otimes c^{n} .
$$


Multiplication of (9) by $c^{l-1} q(c) \otimes 1$ on the left yields $0=-2 c^{l-1} q(c) \otimes c^{n}$ whence $c^{n}=0$.

If we are given (b) then (8) reads

$$
\sum_{k=0}^{n}(-1)^{k}\left(\begin{array}{l}
n \\
k
\end{array}\right) c^{n-k} \otimes c^{k}=0
$$

Multiplication of (10) by $1 \otimes c^{l-1} q(c)$ yields $c^{n} \otimes c^{l-1} q(c)=0$ so again we obtain $c^{n}=0$.

REMARK. Corollary l(b) was first proved for simple rings by Herstein [6] and conjectured for prime rings by Kovacs [10] and Herstein and the proof announced in [13].

The conclusion of Corollary 1 is false for prime rings in general. As an example, let $n=5, R=M_{2}(\mathbf{C})$, the ring of $2 \times 2$ matrices over $\mathbf{C}$, and $a=\left[\begin{array}{cc}1 & 0 \\ 0 & \frac{1+\sqrt{-3}}{2}\end{array}\right]$. One may verify that $(\operatorname{ad} a)^{5}=\operatorname{ad}\left(a^{5}\right)$, but there is no $\lambda \in \mathbf{C}$ such that $(a-\lambda)$ is nilpotent. A more complicated example of the same nature can be constructed as follows:

Let $n=5, R_{j}=M_{2}(\mathbf{C})$ for $j=1,2, \cdots, R=\bigoplus_{j=1}^{\infty} R_{j},\left\{\theta_{j}\right\}_{j=1}^{\infty}$ a collection of distinct real numbers such that $0<\theta_{j}<\pi / 6, z_{j}=e^{i \theta_{j}}$ where $i^{2}=-1, a_{j}=\left(\begin{array}{cc}z_{j} & 0 \\ 0 & z_{j} \frac{1+\sqrt{-3}}{2}\end{array}\right)$, and $a=\oplus a_{j}$. Then $(\operatorname{ad} a)^{5}=\operatorname{ad}\left(a^{5}\right)$ and $a$ has infinitely many distinct eigenvalues.

COROLlARY 2. Let $R$ be a 2-fold transitive algebra of linear transformations on a complex vector space $H$ and suppose $(\operatorname{ad} a)^{n}=\operatorname{ad} b, n>1$, for some $a, b \in R$. Then $a$ is algebraic, and if the minimum polynomial $p(x)$ of a contains a repeated root $\lambda$ (in particular if either $n$ is even or $b=0$ ) then $(a-\lambda)^{[(n+1) / 2]}=0$.

The following corollary shows that the example following Corollary 1 is typical.

COROLlaRY 3. Let $R$ be a 2-fold transitive algebra of linear transformations on a complex vector space $H$, and suppose $(\operatorname{ad} a)^{n}=\operatorname{ad} b$ for some $a, b \in R$ with $n$ odd, $n>1$. If the minimum polynomial $p(x)$ of a has distinct roots $\lambda_{1}, \cdots, \lambda_{k}, k \leq n$, there exist idempotents $p_{1}, \cdots, p_{k}$ with $p_{i} p_{j}=0$ for $i \neq j, \sum_{i=1}^{k} p_{i}=1$ and $a=\sum_{i=1}^{k} \lambda_{i} p_{i}$. If $k>2$ and $0, \lambda, \mu$ are distinct eigenvalues of $a$, then $\lambda^{n}-\mu^{n}=(\lambda-\mu)^{n}$. Conversely, if $R$ is an algebra over a field $F$ of characteristic 0 , with idempotents $p_{1}, \cdots, p_{k}$ such 
that $1=\sum_{l=1}^{k} p_{i}, p_{i} p_{j}=0$ and $\lambda_{1}, \cdots, \lambda_{k}$ distinct elements of $F$ such that $\lambda_{t}^{n}-\lambda_{j}^{n}=\left(\lambda_{i}-\lambda_{j}\right)^{n}, 1 \leq i, j \leq n$, and $n$ odd, then $(\operatorname{ad} a)^{n}=\operatorname{ad}\left(a^{n}\right)$ and $a=\sum_{l=1}^{k} \lambda_{l} p_{l}$.

Proof. Standard linear algebra gives the existence of the $p_{l}$ with the desired properties. Equation (1) of Lemma 1 becomes $\sum_{k=0}^{n}(-1)^{k}\left(\begin{array}{c}n \\ k\end{array}\right) c^{n-k} x c^{k}=\left[c^{n}, x\right]$ for all $x \in R$ where $c=a-\lambda$. Since $n$ is odd we have

$$
0=\sum_{k=1}^{n-1}(-1)^{k}\left(\begin{array}{l}
n \\
k
\end{array}\right) c^{n-k} x c^{k} \quad \text { for all } x \in R
$$

Let $v \neq 0$ be such that $a v=\mu v$. Then $c v=(a-\lambda) v=(\mu-\lambda) v \neq 0$. Hence

$$
\begin{aligned}
0 & =\sum_{k=1}^{n-1}(-1)^{k}\left(\begin{array}{l}
n \\
k
\end{array}\right) c^{n-k} x c^{k} v \\
& =\sum_{k=1}^{n-1}(-1)^{k}\left(\begin{array}{l}
n \\
k
\end{array}\right) c^{n-k}(\mu-\lambda)^{k} x v \quad \text { for all } x \in R .
\end{aligned}
$$

By transitivity,

$$
0=\sum_{k=1}^{n-1}(-1)^{k}\left(\begin{array}{l}
n \\
k
\end{array}\right) c^{n-k}(\mu-\lambda)^{k}=(c-(\mu-\lambda))^{n}-c^{n}+(\mu-\lambda)^{n} .
$$

In terms of $a$ this says $0=(a-\mu)^{n}-(a-\lambda)^{n}+(\mu-\lambda)^{n}$. Therefore, the minimum polynomial $p(x)$ must divide $(x-\mu)^{n}-(x-\lambda)^{n}+$ $(\mu-\lambda)^{n}$. Since 0 is an eigenvalue of $a$ we have $(-\mu)^{n}-(-\lambda)^{n}+(\mu-\lambda)^{n}$ $=0$. The other part of the corollary is a straightforward calculation.

\section{Commuting iterates of derivations.}

THEOREM 3. Let $R$ be a prime ring and let $d$ and $\delta$ be derivations on $R$ such that $\left[d^{n}(x), \delta^{n}(y)\right]=0$ for all $x, y \in R$. Then either $R$ is commutative, or $d^{3 n-1}=0$, or $\delta^{3 n-1}=0$. Furthermore, if $n=1$, and the characteristic of $R \neq 2$, then either $R$ is commutative, or $d=0$, or $\delta=0$.

Proof. Let $W$ be the subring generated by $\left\{d^{n}(x) \mid x \in R\right\}$ and note that $d(W) \subseteq W$. By the Leibniz formula we have

$$
d^{n}(x y)=\sum_{k=0}^{n}\left(\begin{array}{l}
n \\
k
\end{array}\right) d^{k}(x) d^{n-k}(y) \in W \quad \text { for all } x, y \in R .
$$


For $l=1,2, \cdots, n$ we substitute $d^{l-1}(x)$ for $x$ and $d^{2 n-l}(y)$ for $y$ in (1) to obtain

$$
\begin{aligned}
& \sum_{k=0}^{n-l}\left(\begin{array}{l}
n \\
k
\end{array}\right) d^{k+l-1}(x) d^{3 n-k-l}(y) \in W, \\
& \qquad l=1,2, \cdots, n \text { for all } x, y \in R .
\end{aligned}
$$

For $l=n,(2)$ reads

$$
d^{n-1}(x) d^{2 n}(y) \in W,
$$

and for $l=n-1,(2)$ reads

$$
d^{n-2}(x) d^{2 n+1}(y)+\left(\begin{array}{c}
n \\
1
\end{array}\right) d^{n-1}(x) d^{2 n}(y) \in W .
$$

Together (3) and (4) imply that $d^{n-2}(x) d^{2 n+1}(y) \in W$. Continuing in this fashion by comparing successive decreasing values of $l$ from $l=n$ to $l=1$ we have that $x d^{3 n-1}(y) \in W$ for $x, y \in R$. Therefore, $R d^{3 n-1}(y) \subseteq W$ for all $y \in R$. Similarly for all $x, R \delta^{3 n-1}(x) \subseteq V$, the subring generated by $\left\{\delta^{n}(t) \mid t \in R\right\}$. Since $[W, V]=0$ by assumption, the left ideals $R d^{3 n-1}(y)$ and $R \delta^{3 n-1}(x)$ commute for all $x, y \in R$. Without loss of generality we may assume that $d^{3 n-1}(y) \neq 0$ for some $y$ and that $\delta^{3 n-1}(x)$ $\neq 0$ for some $x$. Since we have two commuting non-zero left ideals $R d^{3 n-1}(y)$ and $R \delta^{3 n-1}(x)$ in the prime ring $R, R$ must be commutative.

For $n=1$, if $R$ is not commutative, we may assume $d^{2}=0$. From $d^{2}(x y)=d^{2}(x) y+2 d(x) d(y)+x d^{2}(y)$ we have $d(x) d(y)=0$. In particular, $0=d(x y) d(x)=[d(x) y+x d(y)] d(x)$ whence $d(x) y d(x)=0$ for all $x, y \in R$. Since $R$ is prime it follows that $d(x)=0$ for all $x \in R$.

THEOREM 4. Let $R$ be a prime ring of characteristic $\geq 3 n$ and let $d=\operatorname{ad} b$ be an inner derivation of $R$ satisfying $\left[d^{n}(x), d^{n}(y)\right]=0$ for all $x, y \in R$. Then there exists an element $\lambda$ in the extended centroid of $R$ such that $a=b-\lambda$ satisfies $a^{[(2 n+3) / 3]}=0$.

Proof. We can assume, by [7], that $n>1$. The condition on $d$ clearly extends to the central closure $A=R C+C$ of $R$. By Theorem $3, d^{3 n-1}=0$ and hence by Corollary $1(\mathrm{~b})$ there exists $\lambda \in C$ such that $a=b-\lambda \in A$ satisfies $a^{[3 n / 2]}=0$. If $l$ is the degree of nilpotency of $a$ we have

$$
l \leq \frac{3 n}{2}
$$

and, assuming the theorem to be false, we may also suppose that

$$
l>\frac{2 n+3}{3}
$$


in other words, $3 l-2 n-4 \geq 0$. We then set

$$
p=q=\frac{3 l-2 n-4}{2} \text { if } l \text { is even }
$$

and

$$
p=\frac{3 l-2 n-5}{2}, q=\frac{3 l-2 n-3}{2} \text { if } l \text { is odd. }
$$

In either case $p+q=3 l-2 n-4$, which we wish to view in the form $2 n+p+q=3(l-1)-1$.

Expansion of $\left[d^{n}(x), d^{n}(y)\right]=0$ by the Leibniz formulas, followed by replacement of $x$ by $a x$, yields

$$
0=a^{p}\left[d^{n}(a x), d^{n}(y)\right] a^{q}=g-h
$$

where

$$
g=\sum_{j, k=0}^{n}(-1)^{n-j+k}\left(\begin{array}{l}
n \\
j
\end{array}\right)\left(\begin{array}{l}
n \\
k
\end{array}\right) a^{p+j+1} x a^{2 n-j-k} y a^{q+k}
$$

and

$$
h=\sum_{j, k=0}^{n}(-1)^{n-j+k}\left(\begin{array}{l}
n \\
j
\end{array}\right)\left(\begin{array}{l}
n \\
k
\end{array}\right) a^{p+j} y a^{2 n+1-j-k} x a^{q+k} .
$$

Since $2 n+1+p+q=3(l-1)$ the only possible surviving summand of $g$ occurs when $j+p+1=q+k=l-1$; for $h$ it occurs when $j+p=$ $q+k=l-1$. To see that these terms actually occur it is necessary to show that the $j$ and $k$ thus determined are indeed within the range $0 \leq j$, $k \leq n$. This means verifying

(9) $0 \leq l-p-2 \leq n, \quad 0 \leq l-p-1 \leq n, \quad 0 \leq l-q-1 \leq n$.

We leave it to the reader to check that the various substitutions of (7) and (8) in (9) lead to the following inequalities

$$
0 \leq 2 n-l+i \leq 2 n, \quad i=0,1,2,3
$$

where $i=0,2$ when $l$ is even and $i=1,3$ when $l$ is odd. But the inequalities (10) follow readily from (5) and (6), so that we have established

$$
g=(-1)^{n+p-q+1}\left(\begin{array}{c}
n \\
l-p-2
\end{array}\right)\left(\begin{array}{c}
n \\
l-q-1
\end{array}\right) a^{l-1} x a^{l-1} y a^{l-1}
$$

and

$$
h=(-1)^{n+p-q}\left(\begin{array}{c}
n \\
l-p-1
\end{array}\right)\left(\begin{array}{c}
n \\
l-q-1
\end{array}\right) a^{l-1} y a^{l-1} x a^{l-1}
$$


Setting $x=y$ in (11) and (12), noting that the coefficients of $a^{l-1} x a^{l-1} x a^{l-1}$ in (11) and (12) are of opposite parity, and knowing $g=h$, we may conclude that $a^{l-1} x a^{l-1} x a^{l-1}=0$ for all $x \in A$. This means that the non-zero right ideal $a^{l-1} A$ is nil of bounded degree which in view of [5], Lemma 1.1 provides a contradiction since $A$ is a prime ring.

CoROllaRy 4. Let $R$ be a 2-fold transitive ring of linear transformations on a complex vector space $H$ and let $d=\mathrm{ad} b$ be an inner derivation of $R$ satisfying $\left[d^{n}(x), d^{n}(y)\right]=0$ for all $x, y \in R$. Then there exists $a$ complex scalar $\lambda$ such that $a=b-\lambda$ satisfies $a^{[(2 n+3) / 3]}=0$.

4. Applications. If $A$ is an algebraically irreducible algebra of operators on a complex Banach space $H$ then $A$ is l-fold transitive (since it is irreducible) and hence by [3] it is $m$-fold transitive if $H$ is infinite dimensional or is of dimension at least $m$. In particular, $\mathcal{L}(H)$, the algebra of all bounded linear operators on $H$ is 2-fold transitive so that the previous results apply.

Let $A$ be a $C^{*}$-algebra of operators, containing the identity operator 1 , acting on a complex Hilbert space $H$. Let $R=A^{\prime \prime}$ be the ultraweak closure of $A$ and let $M$ be the universal enveloping von Neumann algebra of $R$. If $\phi$ is any *-representation of $R$ and $\pi$ the natural injection of $R$ into $M$, there exists a normal $*$-representation $\tilde{\phi}$ of $M$ such that $\phi(x)=$ $\tilde{\phi}(\pi(x))$. We have that $\tilde{\phi}(M)=\phi(R)^{\prime \prime}$. If $\phi$ is irreducible, $\tilde{\phi}(M)=\phi(R)^{\prime \prime}$ $=\mathcal{L}\left(H_{\phi}\right)$, the ring of all bounded linear operators on $H_{\phi}$, where $H_{\phi}$ is the representation Hilbert space. If $\tilde{\phi}$ is a normal homomorphism of $M$ onto a von Neumann algebra $N$, there exists a central projection $c \in M$ and a *-isomorphism $\tilde{\psi}$ of $M_{c}$ onto $N$ such that $\tilde{\phi}(x)=\tilde{\psi}(x c)$ for all $x$ in $M$.

THEOREM 5. Let $A$ be a $C^{*}$-algebra of operators acting on a complex Hilbert space $H$, and assume $A$ contains the identity operator 1 . Let $R=A^{\prime \prime}$, the ultraweak closure of $A$, and suppose $(\operatorname{ad} a)^{n}(x)=(\operatorname{ad} b)(x)$ for some $a, b \in A$ and all $x \in A$. If $n$ is odd there exists a central projection $c \in R$ and a central element $z$ in $R$ such that $((a-z) c)^{(n+1) / 2}=0,1-c=$ $\Sigma_{\beta \in B^{\prime}} d_{\beta}$, and $\operatorname{ad}_{\beta}=\sum_{i=1}^{j(\beta)} \lambda_{i}^{\beta} r_{j}^{\beta}$ where the $\lambda_{i}^{\beta}$ are distinct complex numbers, the $r_{j}^{\beta}$ are (not necessarily self-adjoint) orthogonal idempotents, and the $d_{\beta}$ are orthogonal central projections. If $n$ is even there exists a central element $z \in R$ such that $(a-z)^{n / 2}=0$.

Proof. Let $\left\{\phi_{\beta}\right\}_{\beta \in B}$ be a complete set of irreducible representations of $R$. Then $\phi_{\beta}(x)=\tilde{\phi}_{\beta}(\pi(x))$ where $\tilde{\phi}_{\beta}$ is a normal $*$-homomorphism of $M$ on $\mathcal{L}\left(H_{\phi_{\beta}}\right)$. As above, for each $\beta$, there exists a central projection $c_{\beta}$ in $M$ and a $*$-isomorphism $\tilde{\psi}_{\beta}$ of $M_{c_{\beta}}$ on $\mathcal{L}\left(H_{\phi_{\beta}}\right)$ such that $\tilde{\phi}_{\beta}(x)=\tilde{\psi}_{\beta}\left(x c_{\beta}\right)$ for 
all $x \in M$. Now $(\operatorname{ad} a)^{n}(x)=(\operatorname{ad} b)(x)$ for all $x \in A$ implies $(\operatorname{ad} \pi(a))^{n}(x)=(\operatorname{ad} \pi(b))(x)$ for all $x \in M$ so that $\left(\operatorname{ad} \tilde{\phi}_{\beta}(\pi(a))\right)^{n}(x)=$ $\left(\operatorname{ad} \tilde{\phi}_{\beta}(\pi(b))\right)(x)$. Since $\tilde{\phi}_{\beta}$ is irreducible, the results of Theorem 2 and Corollaries 2 and 3 apply. If $n$ is odd then either (i) there exists $\lambda_{\beta} \in \mathbf{C}$ such that $\left(\tilde{\phi}_{\beta}(\pi(a))-\lambda_{\beta}\right)^{(n+1) / 2}=0$ (in the case that the minimum polynomial of $\tilde{\phi}_{\beta}(\pi(a))$ has a repeated root or $\tilde{\phi}_{\beta}(\pi(a))$ is central) or (ii) $\tilde{\phi}_{\beta}(\pi(a))=\sum_{i=1}^{j(\beta)} \lambda_{i}^{\beta} p_{t}^{\beta}$ where the $p_{t}^{\beta}$ are mutually orthogonal idemotents and the $\lambda_{i}^{\beta}$ are distinct. Since $\left\{\phi_{\beta}\right\}_{\beta \in B}$ is complete we have LUB $c_{\beta}=1$. Choose mutually orthogonal central projections $d_{\beta}^{\prime} \in M$ such that $d_{\beta}^{\prime} \leq c_{\beta}$ and $\sum_{p \in B} d_{\beta}^{\prime}=1$. Let $c^{\prime}=\Sigma_{\beta \in B_{1}} d_{\beta}^{\prime}$ where $B_{1}=\{\beta \mid$ (i) holds $\}$. Then $1-c^{\prime}=\Sigma_{\beta \in B \backslash B_{1}=B^{\prime}} d_{\beta}^{\prime}$. If $\beta \in B_{1}$ then $0=\left(\tilde{\phi}_{\beta}(\pi(a))-\lambda_{\beta}\right)^{(n+1) / 2}=$ $\left(\phi_{\beta}(a)-\lambda_{\beta}\right)^{(n+1) / 2}$ so that $\left|\lambda_{\beta}\right| \leq\left\|\phi_{\beta}(a)\right\| \leq\|a\|$. Hence $z^{\prime}=$ $\sum_{\beta \in B_{1}} \lambda_{\beta} d_{\beta}^{\prime} \in Z_{M}$. Moreover, $\beta \in B_{1}$ implies $0=\left(\tilde{\phi}(\pi(a))-\lambda_{\beta}\right)^{(n+1) / 2}$ $=\left(\tilde{\psi}_{\beta}\left(\left(\pi(a)-\lambda_{\beta}\right) c_{\beta}\right)\right)^{(n+1) / 2}$ so that $0=\left(\pi(a)-\lambda_{\beta}\right)^{(n+1) / 2} c_{\beta}$ since $\tilde{\phi}_{\beta}$ is an isomorphism. $\left(\pi(a)-z^{\prime}\right)^{(n+1) / 2} c^{\prime}=\left(\left(\pi(a)-z^{\prime}\right) c^{\prime}\right)^{(n+1) / 2} c_{\beta} d_{\beta}^{\prime}=0$ for each $\beta \in B_{1}$. Similarly if $\beta \in B \backslash B_{1}, \tilde{\phi}_{\beta}(\pi(a))=\sum_{i=1}^{J(\beta)} \lambda_{i}^{\beta} p_{t}^{\beta}=\tilde{\psi}_{\beta}\left(\pi(a) c_{\beta}\right)$ so that $\pi(a) c_{\beta}=\sum_{i=1}^{j(\beta)} \lambda_{i}^{\beta} q_{i}^{\beta}$ and $\pi(a) d_{\beta}^{\prime}=\sum_{i=1}^{j(\beta)} \lambda_{l}^{\beta} q_{l}^{\beta} d_{\beta}^{\prime}$.

Let $i: R \rightarrow R$ be the identity map, $\tilde{i}$ the normal homomorphism of $M$ on $R$ for which $\tilde{i}(\pi(x))=i(x)$ for all $x \in R$. Let $c^{\prime \prime}$ be a central projection in $M$ and $\tilde{j}$ an isomorphism of $M_{c^{\prime \prime}}$ on $R$ such that $\tilde{i}(\pi(x))=$ $\tilde{j}\left(\pi(x) c^{\prime \prime}\right) \cdot \tilde{j}$ induces an isomorphism between $Z_{M_{c^{\prime \prime}}}$ the center of $M_{c_{\tilde{j}}^{\prime \prime}}$ and $Z_{R}$. Hence $c^{\prime} c^{\prime \prime}+\left(1-c^{\prime}\right) c^{\prime \prime}$ which is the identity of $M_{c^{\prime \prime}}$ is sent by $\tilde{j}$ to 1 , the identity of $R$. Let $c=\tilde{j}\left(c^{\prime}, c^{\prime \prime}\right)$ and $z=\tilde{j}\left(z^{\prime}, c^{\prime \prime}\right), d_{\beta}=\tilde{j}\left(d_{\beta}^{\prime} c^{\prime \prime}\right)$, and $r_{j}^{\beta}=\tilde{j}\left(q_{l}^{\beta} d_{\beta}^{\prime} c^{\prime \prime}\right)$.

TheOREM 6. Let $A$ be a $C^{*}$-algebra, $d$ a derivation on $A$ such that $\left[d^{n}(x), d^{n}(y)\right]=0$ for all $x, y \in A$. There exists $s \in R, z \in Z_{R}$ the centre of $R$, such that $d(x)=[s, x]$ for all $x \in A$ and $(s-z)^{[(2 n+3) / 3]}=0$.

Proof. By [16: 4.1.7] there exists such an $s$. Moreover $d$ extends in this way to a derivation on $R$. The result follows from Theorem 4 and an argument as in Theorem 5 .

We finish with a result which does not fit the title of the paper but which contains the same methods in its proof.

LEMMA [See 8: Theorem]. If $R$ is a prime ring not of characteristic 2 and $d: R \rightarrow R$ a derivation, then then either $d=0$ or $\{x \in R \mid[x, d(r)]=0$ for all $r \in R\} \subseteq Z_{R}$, the centre of $R$.

Proof. Let $b \in\{x \in R \mid[x, d(r)]=0$ for all $r \in R\}$. Then $0=$ $[b, d(r)]=((\operatorname{ad} b) \circ d)(r)$ for all $r \in R$. By [15: Theorem 1] either $d=0$ or ad $b=0$. If ad $b=0$ then $[b, r]=0$ for all $r$ so $b \in Z_{R}$. 
THEOREM 7. Let $A$ be a $C^{*}$-algebra with identity $e, R=A^{\prime \prime}$, and $d: A \rightarrow A$ a derivation. There exists a central projection $c \in Z_{R}$ such that $d c(a)=0$ for all $a \in A$, and $\{a(e-c) \mid[a, d(b)](e-c)=0$ for all $b \in$ $A\} \subseteq Z_{A}(e-c)$.

Proof. $d$ extends to a derivation (denoted by $d$ ) from $R$ to $R$. Let $\phi$ be an irreducible representation of $R$ and consider $d_{\phi}: \phi(R) \rightarrow \phi(R)$ given by $d_{\phi}(\phi(r))=\phi(d(r))$. Then $d_{\phi}$ is a derivation on the irreducible algebra $\phi(R)$. By the lemma either $d_{\phi}=0$ or $\left\{\phi(r) \mid\left[\phi(r), d_{\phi}(\phi(s))\right]=0\right.$ for all $s \in R\} \subseteq Z_{\phi(R)}$.

If $\tilde{\psi}, \pi$, and $c$ are as in the beginning of this section,

$$
\begin{aligned}
\{\phi(r) \mid[ & \left.\left.\phi(r), d_{\phi}(\phi(s))\right]=0 \text { for all } s \in R\right\} \\
& =\{\tilde{\psi}(\pi(r) c) \mid[\tilde{\psi}(\pi(r) c), \tilde{\psi}(\pi(d(s)) c)]=0 \text { for all } s \in R\} \\
& =\{\tilde{\psi}(\pi(r) c) \mid[\pi(r) c, \pi(d(s)) c]=0 \text { for all } s \in R\} .
\end{aligned}
$$

Since $\tilde{\psi}$ is a $*$-isomorphism from $M_{c}$ onto $\mathcal{L}\left(H_{\phi}\right)$ it carries centers to centers so that if $\left\{\phi(r) \mid\left[\phi(r), d_{\phi}(\phi(s))\right]=0\right.$ for all $\left.s \in R\right\} \subseteq Z_{\phi(R)}$ we must have $\{\pi(r) c \mid[\pi(r) c, \pi(d(s)) c]=0$ for all $s \in R\} \subseteq Z_{M_{\dot{c}}}$.

Let $\left\{\phi_{\beta}\right\}$ be a complete set of irreducible *-representations of $R$ and $d \phi_{\beta}$ as above. If $d \phi_{\beta}=0$ there exists a central projection $c_{\beta}$ in $M$ such that $0=d \phi_{\beta}\left(\phi_{\beta}(x)\right)=\phi_{\beta}(d(x))=\tilde{\phi}_{\beta}(\pi(d(x)))=\tilde{\psi}_{\beta}\left(\pi(d(x)) c_{\beta}\right)$ so that $\pi(d(x)) c_{\beta}=0$ for all $x \in R$. If $d \phi_{\beta} \neq 0$ there exists $c_{\beta}$ in $M$ such that $\left\{\pi(r) c_{\beta} \mid\left[\pi(r) c_{\beta}, \pi(d(s)) c_{\beta}\right]=0\right.$ for all $\left.s \in R\right\} \subseteq Z_{M_{c_{\beta}}}$

Since $\left\{\phi_{\beta}\right\}$ is complete, $\operatorname{LUB} c_{\beta}=e$, choose mutually orthogonal central projections $c_{\beta}^{\prime}$ in $M$ such that $c_{\beta}^{\prime} \leq c_{\beta}$ and $\Sigma c_{\beta}^{\prime}=e$. Let $c_{0}=\Sigma c_{\beta}^{\prime}$ where the sum is over all $\beta$ such that $\pi(d(x)) c_{\beta}=0$ for all $x \in R$.

Let $i, \tilde{i}$ and $\tilde{j}$ be as above with $c_{1}$ a central projection in $M$ such that $\tilde{j}$ is an isomorphism of $M_{c_{1}}$ on $R$. There exists $c \in Z_{R}$ such that $\tilde{j}\left(c_{0} c_{1}\right)=c$. We have $\tilde{i}\left(c_{0}\right)=c$. Now $0=\pi(d(r)) c_{0}$ for all $r \in R$ so that $0=$ $\tilde{i}\left(\pi(d(r)) c_{0}\right)=d(r) c$ for all $r \in R$. Moreover,

$$
\begin{aligned}
& \left\{\pi(r)\left(e-c_{0}\right) \| \pi(r), \pi(d(s)) \mid\left(e-c_{0}\right)=0 \text { for all } s \in R\right\} \\
& \quad=\left\{\pi(r)\left(e-c_{0}\right) \mid \tilde{i}\left([\pi(r), \pi(d(s))]\left(e-c_{0}\right)\right)=0 \text { for all } s \in R\right\} \\
& \quad=\left\{\pi(r)\left(e-c_{0}\right) \mid[r, d(s)](e-c)=0 \text { for all } s \in R\right\} .
\end{aligned}
$$

Hence $\left\{\pi(r)\left(e-c_{0}\right) \mid[\pi(r), \pi(d(s))]\left(e-c_{0}\right)=0\right.$ for all $\left.s \in R\right\} \subseteq Z_{M_{e-c_{0}}}$ implies $\{r(e-e) \mid[r, d(s)](e-c)=0$ for all $s \in R\} \subseteq \tilde{i}\left(Z_{M_{e-c_{0}}}\right) \stackrel{e-c_{0}}{=}$ $Z_{R_{e-c}}$. 
Finally,

$$
\begin{aligned}
& \{a(e-c) \mid[a, d(b)](e-c)=0 \text { for all } b \in A\} \\
& \quad \subseteq\{r(e-c) \mid[r, d(s)](e-c)=0 \text { for all } s \in R\}
\end{aligned}
$$

by the ultra weak continuity of $d$. Therefore

$$
\begin{aligned}
& \{a(e-c) \mid[a, d(b)](e-c)=0 \text { for all } b \in A\} \\
& \subseteq A(e-c) \cap Z_{R}(e-c)=Z(e-c) .
\end{aligned}
$$

\section{REFERENCES}

1. Jacques Dixmier, $C^{*}$-Algebras, North Holland, New York, 1977.

2. T. S. Erikson, W. S. Martindale, and J. Osborn, Prime nonassociative algebras, Pacific

J. Math., 60 (1975), 49-63.

3. Roger Godement, $A$ theory of spherical functions, I, Trans. Amer. Math. Soc., 73 (1952), 496-556.

4. I. N. Herstein, Noncommutative Rings, Carus Monograph, 15, 1968.

5. __ _ Topics in Ring Theory, University of Chicago Press, 1969.

6 . Sui commutator degli anelli semplici, Rendiconte del Seminario Matematico e Fisico di Milano, Vol. XXXIII, 1963.

7. _ A note on derivations, Canad. Math. Bull., 00 (1977).

8. _ _ A note on derivations II, Canad. Math. Bull. (to appear).

$9 . \quad$, Rings with Involution, University of Chicago Press, 1976.

10. A. Kovacs, Nilpotent derivations, Technion Preprint Series, No. NT-453.

11. W. S. Martindale, 3rd, Lie isomorphisms of prime rings, Trans. Amer. Math. Soc., 142 (1969), 437-455.

12. __ Prime rings with involution and generalized polynomial identities, J. Algebra, 22 (1972), 502-516.

13. Notices of the American Math. Soc.

14. C. R. Miers, Lie homomorphisms of operator algebras, Pacific J. Math., 38 (1971), 717-737.

15. E. C. Posner, Derivations in prime rings, Proc. Amer. Math. Soc., 8 (1957), 1093-1100.

16. S. Sakai, $C^{*}$-Algebras and $W^{*}$-Algebras, Springer-Verlag, New York, 1971.

Received October 31, 1980 and in revised form February 10, 1982. Partially supported by NSERC Grant 7682.

UNIVERSITY OF VICTORIA

Victoria, British Columbia, Canada V8W 2Y2 


\section{PACIFIC JOURNAL OF MATHEMATICS \\ EDITORS}

DONALD BABBITT (Managing Editor)

University of California

Los Angeles, CA 90024

\section{Hugo Rossi}

University of Utah

Salt Lake City, UT 84112

C. C. Moore and Arthur Ogus

University of California

Berkeley, CA 94720
J. DugundiI

Department of Mathematics

University of Southern California

Los Angeles, CA 90089-1113

R. FINN and H. SAMELSON

Stanford University

Stanford, CA 94305

ASSOCIATE EDITORS
R. ARens
E. F. BECKENBACH
B. H. NeumanN
F. WOLF
K. YoshidA (1906-1982)

\section{SUPPORTING INSTITUTIONS}

UNIVERSITY OF ARIZONA

UNIVERSITY OF BRITISH COLUMBIA

CALIFORNIA INSTITUTE OF TECHNOLOGY

UNIVERSITY OF CALIFORNIA

MONTANA STATE UNIVERSITY

UNIVERSITY OF NEVADA, RENO

NEW MEXICO STATE UNIVERSITY

OREGON STATE UNIVERSITY
UNIVERSITY OF OREGON

UNIVERSITY OF SOUTHERN CALIFORNIA

STANFORD UNIVERSITY

UNIVERSITY OF HAWAII

UNIVERSITY OF TOKYO

UNIVERSITY OF UTAH

WASHINGTON STATE UNIVERSITY

UNIVERSITY OF WASHINGTON 


\section{Pacific Journal of Mathematics}

Vol. 104, No. 1

May, 1983

Nestor Edgardo Aguilera and Eleonor Ofelia Harboure de Aguilera, On

the search for weighted norm inequalities for the Fourier transform $\ldots \ldots .1$

Jin Akiyama, Frank Harary and Phillip Arthur Ostrand, A graph and its complement with specified properties. VI. Chromatic and achromatic numbers ......................................... 15

Bing Ren Li, The perturbation theory for linear operators of discrete type . . . 29

Peter Botta, Stephen J. Pierce and William E. Watkins, Linear

transformations that preserve the nilpotent matrices .............. 39

Frederick Ronald Cohen, Ralph Cohen, Nicholas J. Kuhn and Joseph

Alvin Neisendorfer, Bundles over configuration spaces .......... 47

Luther Bush Fuller, Trees and proto-metrizable spaces . . . . . . . . . . 55

Giovanni P. Galdi and Salvatore Rionero, On the best conditions on the

gradient of pressure for uniqueness of viscous flows in the whole space . . 77

John R. Graef, Limit circle type results for sublinear equations $\ldots \ldots \ldots \ldots 85$

Andrzej Granas, Ronald Bernard Guenther and John Walter Lee,

Topological transversality. II. Applications to the Neumann problem for

$y^{\prime \prime}=f\left(t, y, y^{\prime}\right) \ldots \ldots \ldots \ldots \ldots \ldots \ldots \ldots \ldots \ldots \ldots \ldots \ldots \ldots \ldots . \ldots 5$

Richard Howard Hudson and Kenneth S. Williams, Extensions of

theorems of Cunningham-Aigner and Hasse-Evans . . . . . . . . . . . 111

John Francis Kurtzke, Jr., Centralizers of irregular elements in reductive algebraic groups

James F. Lawrence, Lopsided sets and orthant-intersection by convex

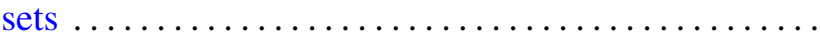

Åsvald Lima, G. H. Olsen and U. Uttersrud, Intersections of $M$-ideals and

$G$-spaces

Wallace Smith Martindale, III and C. Robert Miers, On the iterates of derivations of prime rings

Thomas H. Pate, Jr, A characterization of a Neuberger type iteration procedure that leads to solutions of classical boundary value problems

Carl L. Prather and Ken Shaw, Zeros of successive iterates of multiplier-sequence operators

Billy E. Rhoades, The fine spectra for weighted mean operators

Rudolf J. Taschner, A general version of van der Corput's difference theorem

Johannes A. Van Casteren, Operators similar to unitary or selfadjoint ones 